\title{
FILTERING OF MULTIDIMENSIONAL STATIONARY SEQUENCES WITH MISSING OBSERVATIONS
}

\begin{abstract}
The problem of mean-square optimal linear estimation of linear functionals which depend on the unknown values of a multidimensional stationary stochastic sequence is considered. Estimates are based on observations of the sequence with an additive stationary stochastic noise sequence at points which do not belong to some finite intervals of a real line. Formulas for calculating the mean-square errors and the spectral characteristics of the optimal linear estimates of the functionals are proposed under the condition of spectral certainty, where spectral densities of the sequences are exactly known. The minimax (robust) method of estimation is applied in the case where spectral densities are not known exactly while some sets of admissible spectral densities are given. Formulas that determine the least favorable spectral densities and minimax spectral characteristics are proposed for some special sets of admissible densities.

Key words and phrases: stationary sequence, minimax-robust estimate, mean square error, least favorable spectral density, minimax spectral characteristic.
\end{abstract}

Taras Shevchenko National University, 64/13 Volodymyrska str., 01601, Kyiv, Ukraine

E-mail: omasyutka@gmail.com (Masyutka O.Yu.), moklyachuk@gmail.com (Moklyachuk M.P.), marysidei4@gmail.com (Sidei M.I.)

\section{INTRODUCTION}

The problem of estimation of the unknown values of stochastic processes is of constant interest in the theory and applications of stochastic processes. The formulation of the estimation problems (interpolation, extrapolation and filtering) for stationary stochastic sequences with known spectral densities and reducing these problems to the corresponding problems of the theory of functions belongs to Kolmogorov [17]. Effective methods of solution of the estimation problems for stationary stochastic sequences and processes were developed by Wiener [41] and Yaglom [42,43]. Further results are described in the books by Rozanov [38], Hannan [12], Box et al. [3], Brockwell and Davis [4]. The crucial assumption of most of the methods developed for estimating the unobserved values of stochastic processes is that the spectral densities of the involved stochastic processes are exactly known. In practice, however, complete information on the spectral densities is impossible in most cases. In this situation one finds parametric or nonparametric estimates of the unknown spectral densities and then apply one of the traditional estimation methods provided that the selected spectral densities are true. This procedure can result in significant increasing of the value of the error of estimate as Vastola and Poor [40] have demonstrated with the help of some examples. To avoid this effect one can search estimates which are optimal for all densities from a certain given class of admissible spectral densities. These estimates are called minimax since they minimize the maximum value of the error of 
estimates. The paper by Grenander [11] was the first one where this approach to extrapolation problem for stationary processes was proposed. Several models of spectral uncertainty and minimax-robust methods of data processing can be found in the survey paper by Kassam and Poor [16]. Franke [7, 8], Franke and Poor [9] investigated the minimax extrapolation and filtering problems for stationary sequences with the help of convex optimization methods. This approach makes it possible to find equations that determine the least favorable spectral densities for some classes of admissible densities.

In the papers by Moklyachuk $[23,25,26]$ results of investigation of the extrapolation, interpolation and filtering problems for functionals which depend on the unknown values of stationary processes and sequences are described. The problem of estimation of functionals which depend on the unknown values of multivariate stationary stochastic processes is the aim of the papers by Moklyachuk and Masyutka [28,29]. In the book by Moklyachuk and Golichenko [27] results of investigation of the interpolation, extrapolation and filtering problems for periodically correlated stochastic sequences are proposed. In their papers Luz and Moklyachuk [18-22] deal with the problems of estimation of functionals which depend on the unknown values of stochastic sequences with stationary increments. Prediction problem for stationary sequences with missing observations is investigated in papers by Bondon [1,2], Cheng, Miamee and Pourahmadi [5], Cheng and Pourahmadi [6], Kasahara, Pourahmadi and Inoue [15], Pourahmadi, Inoue and Kasahara [35], Pelagatti [34]. In papers by Moklyachuk and Sidei [30-33] an approach is developed to investigation of the interpolation, extrapolation and filtering problems for stationary stochastic sequences with missing observations.

In this paper we investigate the problem of the mean-square optimal estimation of the functional $A \vec{\xi}=\sum_{j \in Z^{S}} \vec{a}(j)^{\top} \vec{\xi}(-j)$ which depends on the unknown values of a multidimensional stationary sequence $\{\vec{\xi}(j), j \in \mathbb{Z}\}$ from the observations of the sequence $\{\vec{\xi}(j)+\vec{\eta}(j)\}$ at points $j \in \mathbb{Z}_{-} \backslash S$, where $\{\vec{\eta}(j), j \in \mathbb{Z}\}$ is uncorrelated with $\{\vec{\xi}(j), j \in \mathbb{Z}\}$ multidimensional stationary sequence, $S=\bigcup_{l=1}^{S}\left\{-\left(M_{l}+N_{l}\right), \ldots,-M_{l}\right\}, Z^{S}=\{1,2, \ldots\} \backslash S^{+}, S^{+}=\bigcup_{l=1}^{S}\left\{M_{l}, \ldots, M_{l}+N_{l}\right\}$, $M_{0}=0, N_{0}=0$. The problem is investigated in the case where both spectral densities of the sequences $\{\vec{\xi}(j), j \in \mathbb{Z}\}$ and $\{\vec{\eta}(j), j \in \mathbb{Z}\}$ are known. In this case we derive formulas for calculating the spectral characteristic and the mean-square error of the optimal estimate using the method of projection in the Hilbert space of random variables with finite second moments proposed by Kolmogorov (see, for example, selected works by Kolmogorov [17]). In the case of spectral uncertainty, where the spectral densities of the sequences are not exactly known while a set of admissible spectral densities is given, the minimax method is applied. Formulas that determine the least favorable spectral densities and the minimax-robust spectral characteristics of the optimal estimates of the functional are proposed for some specific classes of admissible spectral densities.

\section{HiLbERT SPACE PROJECTION METHOD OF FILTERING}

Consider multidimensional stationary stochastic sequences $\vec{\xi}(j)=\left\{\xi_{k}(j)\right\}_{k=1}^{T}, j \in \mathbb{Z}$, and $\vec{\eta}(j)=\left\{\eta_{k}(j)\right\}_{k=1}^{T}, j \in \mathbb{Z}$, with absolutely continuous spectral functions and correlation func- 
tions of the form

$$
\begin{aligned}
& R_{\tilde{\xi}}(n)=E \vec{\xi}(j+n)(\vec{\xi}(j))^{*}=\frac{1}{2 \pi} \int_{-\pi}^{\pi} e^{i n \lambda} F(\lambda) d \lambda, \\
& R_{\eta}(n)=E \vec{\eta}(j+n)(\vec{\eta}(j))^{*}=\frac{1}{2 \pi} \int_{-\pi}^{\pi} e^{i n \lambda} G(\lambda) d \lambda,
\end{aligned}
$$

where $F(\lambda)=\left\{f_{k l}(\lambda)\right\}_{k, l=1}^{T}, G(\lambda)=\left\{g_{k l}(\lambda)\right\}_{k, l=1}^{T}$ are the spectral densities of the sequences $\{\vec{\xi}(j), j \in \mathbb{Z}\}$ and $\{\vec{\eta}(j), j \in \mathbb{Z}\}$ respectively. We will suppose that the spectral densities $F(\lambda)$ and $G(\lambda)$ satisfy the minimality condition

$$
\int_{-\pi}^{\pi} \operatorname{Tr}(F(\lambda)+G(\lambda))^{-1} d \lambda<\infty
$$

This condition is necessary and sufficient in order that the error-free filtering of unknown values of the sequences is impossible (see, for example, Rozanov [38]).

The stationary stochastic sequences $\{\vec{\xi}(j)\}$ and $\{\vec{\eta}(j)\}$ admit the following spectral decomposition (see, for example, Gikhman and Skorokhod [10]; Karhunen [14])

$$
\xi(j)=\int_{-\pi}^{\pi} e^{i j \lambda} Z_{\xi}(d \lambda), \quad \eta(j)=\int_{-\pi}^{\pi} e^{i j \lambda} Z_{\eta}(d \lambda),
$$

where $Z_{\xi}(d \lambda)$ and $Z_{\eta}(d \lambda)$ are orthogonal stochastic measures defined on $[-\pi, \pi)$ such that the following relations hold true

$$
\begin{aligned}
& E Z_{\xi}\left(\Delta_{1}\right)\left(Z_{\xi}\left(\Delta_{2}\right)\right)^{*}=\frac{1}{2 \pi} \int_{\Delta_{1} \cap \Delta_{2}} F(\lambda) d \lambda, \\
& E Z_{\eta}\left(\Delta_{1}\right)\left(Z_{\eta}\left(\Delta_{2}\right)\right)^{*}=\frac{1}{2 \pi} \int_{\Delta_{1} \cap \Delta_{2}} G(\lambda) d \lambda .
\end{aligned}
$$

Suppose that we have observations of the sequence $\{\vec{\xi}(j)+\vec{\eta}(j)\}$ at points $j \in \mathbb{Z}_{-} \backslash S$, where $S=\bigcup_{l=1}^{S}\left\{-\left(M_{l}+N_{l}\right), \ldots,-M_{l}\right\}$. The problem is to find the mean-square optimal linear estimate of the functional

$$
A \vec{\xi}=\sum_{j \in Z^{S}} \vec{a}(j)^{\top} \vec{\xi}(-j),
$$

which depends on the unknown values of the sequence $\{\vec{\xi}(j)\}, Z^{S}=\{1,2, \ldots\} \backslash S^{+}$, $S^{+}=\bigcup_{l=1}^{S}\left\{M_{l}, \ldots, M_{l}+N_{l}\right\}$.

Suppose that coefficients $\{\vec{a}(j), j=0,1, \ldots\}$ defining the functional $A \vec{\xi}$ satisfy the condition

$$
\sum_{j \in Z^{S}} \sum_{k=1}^{T}\left|a_{k}(j)\right|<\infty \text {. }
$$

This condition ensures that the functional $A \vec{\xi}$ has a finite second moment, since

$$
E|A \xi|^{2} \leq \max _{1 \leq k \leq T} E\left|\xi_{k}(0)\right|^{2}\left(\sum_{j \in Z^{S}} \sum_{k=1}^{T}\left|a_{k}(j)\right|\right)^{2}
$$


It follows from the spectral decomposition of the sequence $\{\vec{\xi}(j)\}$ that the functional $A \vec{\xi}$ can be represented in the following form

$$
A \vec{\xi}=\int_{-\pi}^{\pi}\left(A\left(e^{i \lambda}\right)\right)^{\top} Z_{\tilde{\zeta}}(d \lambda), \quad A\left(e^{i \lambda}\right)=\sum_{j \in Z^{S}} \vec{a}(j) e^{-i j \lambda} .
$$

Consider values $\xi_{k}(j), k=1, \ldots, T ; j \in \mathbb{Z}$ and $\eta_{k}(j), k=1, \ldots, T ; j \in \mathbb{Z}$ as elements of the Hilbert space $H=L_{2}(\Omega, \mathcal{F}, P)$ generated by random variables $\xi$ with zero mathematical expectations, $E \xi=0$, finite variations, $E|\xi|^{2}<\infty$, and the inner product $(\xi, \eta)=E \xi \bar{\eta}$. Denote by $H^{s}(\xi+\eta)$ the closed linear subspace generated by elements $\left\{\xi_{k}(j)+\eta_{k}(j): j \in \mathbb{Z}_{-} \backslash S, k=\right.$ $\overline{1, T}\}$ in the Hilbert space $H$.

Denote by $L_{2}(F+G)$ the Hilbert space of vector-valued functions $\vec{a}(\lambda)=\left\{a_{k}(\lambda)\right\}_{k=1}^{T}$ such that

$$
\int_{-\pi}^{\pi} \vec{a}(\lambda)^{\top}(F(\lambda)+G(\lambda)) \overline{\vec{a}(\lambda)} d \lambda<\infty .
$$

Denote by $L_{2}^{s}(F+G)$ the subspace of $L_{2}(F+G)$ generated by functions of the form

$$
e^{i n \lambda} \delta_{k}, \delta_{k}=\left\{\delta_{k l}\right\}_{l=1}^{T}, k=1, \ldots, T, n \in Z_{-} \backslash S,
$$

where $\delta_{k l}$ are Kronecker symbols.

The mean square optimal linear estimate $\hat{A} \vec{\xi}$ of the functional $A \vec{\xi}$ from observations of the sequence $\{\vec{\xi}(j)+\vec{\eta}(j)\}$ can be represented in the form

$$
\hat{A} \vec{\xi}=\int_{-\pi}^{\pi}\left(h\left(e^{i \lambda}\right)\right)^{\top}\left(Z_{\xi}(d \lambda)+Z_{\eta}(d \lambda)\right),
$$

where $h\left(e^{i \lambda}\right)=\left\{h_{k}\left(e^{i \lambda}\right)\right\}_{k=1}^{T} \in L_{2}^{S}(F+G)$ is the spectral characteristic of the estimate.

The mean square error $\Delta(h ; F, G)$ of the estimate $\hat{A} \vec{\xi}$ is given by the formula

$$
\begin{aligned}
\Delta(h ; F, G)=E|A \vec{\xi}-\hat{A} \vec{\xi}|^{2} & =\frac{1}{2 \pi} \int_{-\pi}^{\pi}\left(A\left(e^{i \lambda}\right)-h\left(e^{i \lambda}\right)\right)^{\top} F(\lambda) \overline{\left(A\left(e^{i \lambda}\right)-h\left(e^{i \lambda}\right)\right)} d \lambda \\
& +\frac{1}{2 \pi} \int_{-\pi}^{\pi}\left(h\left(e^{i \lambda}\right)\right)^{\top} G(\lambda) \overline{\left(h\left(e^{i \lambda}\right)\right)} d \lambda .
\end{aligned}
$$

The Hilbert space projection method proposed by Kolmogorov [17] makes it possible to find the spectral characteristic $h\left(e^{i \lambda}\right)$ and the mean square error $\Delta(h ; F, G)$ of the optimal linear estimate of the functional $A \vec{\xi}$ in the case where spectral densities $F(\lambda)$ and $G(\lambda)$ of the sequences are exactly known and the minimality condition (1) is satisfied. According to this method the optimal estimation of the functional $A \vec{\xi}$ is a projection of the element $A \vec{\xi}$ of the space $H$ on the space $H^{s}(\xi+\eta)$. It can be found from the following conditions:

$$
\begin{aligned}
& \text { 1) } \hat{A} \vec{\xi} \in H^{S}(\xi+\eta), \\
& \text { 2) } A \vec{\xi}-\hat{A} \vec{\xi} \perp H^{S}(\xi+\eta) .
\end{aligned}
$$

It follows from the second condition that the spectral characteristic $h\left(e^{i \lambda}\right)$ for any $j \in \mathbb{Z}_{-} \backslash S$ satisfies the equation

$$
\frac{1}{2 \pi} \int_{-\pi}^{\pi}\left(A\left(e^{i \lambda}\right)-h\left(e^{i \lambda}\right)\right)^{\top} F(\lambda) e^{-i j \lambda} d \lambda-\frac{1}{2 \pi} \int_{-\pi}^{\pi}\left(h\left(e^{i \lambda}\right)\right)^{\top} G(\lambda) e^{-i j \lambda} d \lambda=\overrightarrow{0} .
$$


The last relation is equivalent to equations

$$
\frac{1}{2 \pi} \int_{-\pi}^{\pi}\left[\left(A\left(e^{i \lambda}\right)\right)^{\top} F(\lambda)-\left(h\left(e^{i \lambda}\right)\right)^{\top}(F(\lambda)+G(\lambda))\right] e^{-i j \lambda} d \lambda=\overrightarrow{0}, j \in \mathbb{Z}_{-} \backslash S .
$$

Hence the function $\left[\left(A\left(e^{i \lambda}\right)\right)^{\top} F(\lambda)-\left(h\left(e^{i \lambda}\right)\right)^{\top}(F(\lambda)+G(\lambda))\right]$ is of the form

$$
\left(A\left(e^{i \lambda}\right)\right)^{\top} F(\lambda)-\left(h\left(e^{i \lambda}\right)\right)^{\top}(F(\lambda)+G(\lambda))=\left(C\left(e^{i \lambda}\right)\right)^{\top},
$$

where

$$
C\left(e^{i \lambda}\right)=\sum_{j \in S} \vec{c}(j) e^{i j \lambda}+\sum_{j=0}^{\infty} \vec{c}(j) e^{i j \lambda}
$$

Here $\vec{c}(j), j \in U=S \cup\{0,1,2, \ldots\}$ are unknown coefficients that we have to find.

From the last relation we deduce that the spectral characteristic of the optimal linear estimate $\hat{A} \vec{\xi}$ is of the form

$$
\left(h\left(e^{i \lambda}\right)\right)^{\top}=\left(A\left(e^{i \lambda}\right)\right)^{\top} F(\lambda)(F(\lambda)+G(\lambda))^{-1}-\left(C\left(e^{i \lambda}\right)\right)^{\top}(F(\lambda)+G(\lambda))^{-1} .
$$

It follows from the first condition, $\hat{A} \vec{\xi} \in H^{s}(\xi+\eta)$, which determine the optimal linear estimate of the functional $A \vec{\xi}$, that the Fourier coefficients of the function $h\left(e^{i \lambda}\right)$ are equal to zero for $k \in U$, namely

$$
\frac{1}{2 \pi} \int_{-\pi}^{\pi}\left(\left(A\left(e^{i \lambda}\right)\right)^{\top} F(\lambda)(F(\lambda)+G(\lambda))^{-1}-\left(C\left(e^{i \lambda}\right)\right)^{\top}(F(\lambda)+G(\lambda))^{-1}\right) e^{-i k \lambda} d \lambda=\overrightarrow{0}, k \in U .
$$

We will use the last equality to find equations which determine the unknown coefficients $\vec{c}(j), j \in U$. After disclosing the brackets we get the relation

$$
\begin{aligned}
& \sum_{j \in Z^{S}} \vec{a}(j)^{\top} \frac{1}{2 \pi} \int_{-\pi}^{\pi} F(\lambda)(F(\lambda)+G(\lambda))^{-1} e^{-i(k+j) \lambda} d \lambda-\sum_{j \in S} \vec{c}(j)^{\top} \frac{1}{2 \pi} \int_{-\pi}^{\pi}(F(\lambda) \\
& +G(\lambda))^{-1} e^{-i(k-j) \lambda} d \lambda-\sum_{j=0}^{\infty} \vec{c}(j)^{\top} \frac{1}{2 \pi} \int_{-\pi}^{\pi}(F(\lambda)+G(\lambda))^{-1} e^{-i(k-j) \lambda} d \lambda=\overrightarrow{0}, k \in U .
\end{aligned}
$$

For the functions

$$
(F(\lambda)+G(\lambda))^{-1}, \quad F(\lambda)(F(\lambda)+G(\lambda))^{-1}, \quad F(\lambda)(F(\lambda)+G(\lambda))^{-1} G(\lambda)
$$

we introduce the Fourier coefficients

$$
\begin{gathered}
B(k, j)=\frac{1}{2 \pi} \int_{-\pi}^{\pi}(F(\lambda)+G(\lambda))^{-1} e^{-i(k-j) \lambda} d \lambda, \\
R(k, j)=\frac{1}{2 \pi} \int_{-\pi}^{\pi} F(\lambda)(F(\lambda)+G(\lambda))^{-1} e^{-i(k+j) \lambda} d \lambda,
\end{gathered}
$$




$$
Q(k, j)=\frac{1}{2 \pi} \int_{-\pi}^{\pi} F(\lambda)(F(\lambda)+G(\lambda))^{-1} G(\lambda) e^{-i(k-j) \lambda} d \lambda .
$$

Using the introduced notations we can verify that the equality (2) is equivalent to the following system of equations:

$$
\sum_{j \in Z^{S}} R(k, j) \vec{a}(j)=\sum_{j \in S} B(k, j) \vec{c}(j)+\sum_{j=0}^{\infty} B(k, j) \vec{c}(j), \quad k \in U .
$$

Let us introduce notations $\vec{a}(j)=\overrightarrow{0}, j \in S, \vec{a}(0)=\overrightarrow{0}$ and $\vec{a}(j)=\overrightarrow{0}, j \in S^{+}$. Thus, we can write

$$
\sum_{j \in U} R(k, j) \vec{a}(j)=\sum_{j \in S} B(k, j) \vec{c}(j)+\sum_{j=0}^{\infty} B(k, j) \vec{c}(j), \quad k \in U
$$

The last equations can be rewritten in the following form

$$
\mathbf{R} \overrightarrow{\mathbf{a}}=\mathbf{B} \overrightarrow{\mathbf{c}},
$$

where $\overrightarrow{\mathbf{c}}$ is a vector constructed from the unknown coefficients $\vec{c}(j), j \in U$, vector $\overrightarrow{\mathbf{a}}$ has the same with the vector $\overrightarrow{\mathbf{c}}$ dimension, it is of the form

$$
\overrightarrow{\mathbf{a}}^{\top}=\left(\overrightarrow{0}_{0}^{\top}, \vec{a}_{1}^{\top}, \overrightarrow{0}_{1}^{\top}, \vec{a}_{2}^{\top}, \overrightarrow{0}_{2}^{\top}, \ldots \vec{a}_{i}^{\top}, \overrightarrow{0}_{i}^{\top}, \ldots, \vec{a}_{s}^{\top}, \overrightarrow{0}_{s}^{\top}, \vec{a}_{s+1}^{\top}\right),
$$

where $\overrightarrow{0}_{0}$ is the vector which consists of $(|S|+1) T$ zeros, where $|S|=\sum_{k=1}^{S}\left(N_{k}+1\right)$ is the amount of missing values, vectors $\overrightarrow{0}_{i}, i=1,2, \ldots, s$, consist of $\left(N_{i}+1\right) T$ zeros, vectors

$$
\begin{gathered}
\vec{a}_{1}^{\top}=\left(\vec{a}(1)^{\top}, \ldots, \vec{a}\left(M_{1}-1\right)^{\top}\right), \\
\vec{a}_{i}^{\top}=\left(\vec{a}\left(M_{i-1}+N_{i-1}+1\right)^{\top}, \ldots, \vec{a}\left(M_{i}-1\right)^{\top}\right), \quad i=2, \ldots, s, \\
\vec{a}_{s+1}^{\top}=\left(\vec{a}\left(M_{s}+N_{s}+1\right)^{\top}, \vec{a}\left(M_{s}+N_{s}+2\right)^{\top}, \ldots\right),
\end{gathered}
$$

are constructed from the coefficients that determine the functional $A \vec{\xi}$.

Here $\mathbf{B}$ is a linear operator in the space $\ell_{2}$ which is defined by the matrix

$$
B=\left(\begin{array}{ccccc}
B_{s, s} & B_{s, s-1} & \ldots & B_{s, 1} & B_{s, n} \\
B_{s-1, s} & B_{s-1, s-1} & \ldots & B_{s-1,1} & B_{s-1, n} \\
\vdots & \vdots & \ddots & \vdots & \vdots \\
B_{1, s} & B_{1, s-1} & \ldots & B_{1,1} & B_{1, n} \\
B_{n, s} & B_{n, s-1} & \ldots & B_{n, 1} & B_{n, n}
\end{array}\right)
$$

where elements in the last column and the last row are compound matrices constructed from the block-matrices

$$
\begin{aligned}
& B_{l, n}(k, j)=B(k, j), l=1,2, \ldots, s, k=-M_{l}-N_{l}, \ldots,-M_{l}, j=0,1,2, \ldots, \\
& B_{n, m}(k, j)=B(k, j), m=1,2, \ldots, s, k=0,1,2, \ldots, j=-M_{m}-N_{m}, \ldots,-M_{m}, \\
& B_{n, n}(k, j)=B(k, j), k, j=0,1,2, \ldots
\end{aligned}
$$


and other elements of matrix $B$ are the compound matrices with elements of the form

$$
\begin{array}{r}
B_{l, m}(j, k)=B(k, j), \quad l, m=1,2, \ldots, s, \\
k=-M_{l}-N_{l}, \ldots,-M_{l}, \quad j=-M_{m}-N_{m}, \ldots,-M_{m} .
\end{array}
$$

The linear operator $\mathbf{R}$ in the space $\ell_{2}$ is defined by the corresponding matrix in the same manner.

The unknown coefficients $\vec{c}(k), k \in U$, which are defined by the equations (3), can be calculated by the formula

$$
\vec{c}(k)=\left(\mathbf{B}^{-1} \mathbf{R} \overrightarrow{\mathbf{a}}\right)(k),
$$

where $\left(\mathbf{B}^{-1} \mathbf{R} \overrightarrow{\mathbf{a}}\right)(k)$ is the $k$-th component of the vector $\mathbf{B}^{-1} \mathbf{R} \overrightarrow{\mathbf{a}}$. (see paper by Salehi [39] for more details).

The formula for calculating the spectral characteristic $h\left(e^{i \lambda}\right)$ of the estimate $\hat{A} \vec{\xi}$ is of the form

$$
\begin{aligned}
\left(h\left(e^{i \lambda}\right)\right)^{\top}=\left(A\left(e^{i \lambda}\right)\right)^{\top} F(\lambda)(F(\lambda) & +G(\lambda))^{-1} \\
& -\left(\sum_{k \in U}\left(\mathbf{B}^{-1} \mathbf{R} \overrightarrow{\mathbf{a}}\right)(k) e^{i k \lambda}\right)^{\top}(F(\lambda)+G(\lambda))^{-1} .
\end{aligned}
$$

The mean square error of the estimate $\hat{A} \vec{\xi}$ can be calculated by the formula

$$
\begin{aligned}
\Delta(F, G)=E|A \vec{\xi}-\hat{A} \vec{\xi}|^{2}= & \frac{1}{2 \pi} \int_{-\pi}^{\pi}\left(r_{G}(\lambda)\right)^{\top} F(\lambda) \overline{r_{G}(\lambda)} d \lambda \\
& +\frac{1}{2 \pi} \int_{-\pi}^{\pi}\left(r_{F}(\lambda)\right)^{\top} G(\lambda) \overline{r_{F}(\lambda)} d \lambda=\left\langle\mathbf{R} \overrightarrow{\mathbf{a}}, \mathbf{B}^{-1} \mathbf{R} \overrightarrow{\mathbf{a}}\right\rangle+\langle\mathbf{Q} \overrightarrow{\mathbf{a}}, \overrightarrow{\mathbf{a}}\rangle,
\end{aligned}
$$

where

$$
\begin{aligned}
& \left(r_{F}(\lambda)\right)^{\top}=\left(\left(A\left(e^{i \lambda}\right)\right)^{\top} F(\lambda)-\left(\sum_{k \in U}\left(\mathbf{B}^{-1} \mathbf{R} \overrightarrow{\mathbf{a}}\right)(k) e^{i k \lambda}\right)^{\top}\right)(F(\lambda)+G(\lambda))^{-1}, \\
& \left(r_{G}(\lambda)\right)^{\top}=\left(\left(A\left(e^{i \lambda}\right)\right)^{\top} G(\lambda)+\left(\sum_{k \in U}\left(\mathbf{B}^{-1} \mathbf{R} \overrightarrow{\mathbf{a}}\right)(k) e^{i k \lambda}\right)^{\top}\right)(F(\lambda)+G(\lambda))^{-1},
\end{aligned}
$$

and $\mathbf{Q}$ is the linear operator in the space $\ell_{2}$ defined by matrix with coefficients $Q(k, j), k, j \in U$ in the same way as the operator $\mathbf{B}$ is defined.

Let us summarize the obtained results and present them in the form of a theorem.

Theorem 1. Let $\{\vec{\xi}(j)\}$ and $\{\vec{\eta}(j)\}$ be uncorrelated multidimensional stationary sequences with spectral densities $F(\lambda)$ and $G(\lambda)$ which satisfy the minimality condition (1). The spectral characteristic $h\left(e^{i \lambda}\right)$ and the mean square error $\Delta(F, G)$ of the optimal linear estimate of the functional $A \vec{\xi}$ which depends on the unknown values of the sequence $\vec{\xi}(j)$ based on observations of the sequence $\{\vec{\xi}(j)+\vec{\eta}(j)\}$ at points $j \in \mathbb{Z}_{-} \backslash S$ can be calculated by formulas (4), (5). 
Consider the problem of the mean-square optimal linear estimation of the functional $A \vec{\xi}$, which depends on the unknown values of the sequence $\{\vec{\xi}(j)\}$ from observations of the sequence $\{\vec{\xi}(j)+\vec{\eta}(j)\}$ at points $j \in \mathbb{Z}_{-} \backslash S, S=\{-(M+N), \ldots,-M\}, Z^{S}=\{1,2, \ldots\} \backslash S^{+}$, $S^{+}=\{M, \ldots, M+N\}$.

From Theorem 1 the following corollary can be derived for this problem.

Corollary 1. Let $\{\vec{\xi}(j)\}$ and $\{\vec{\eta}(j)\}$ be uncorrelated multidimensional stationary sequences with spectral densities $F(\lambda)$ and $G(\lambda)$ which satisfy the minimality condition (1). The spectral characteristic $h\left(e^{i \lambda}\right)$ and the mean square error $\Delta(F, G)$ of the optimal linear estimate of the functional $A \vec{\xi}$ which depends on the unknown values of the sequence $\vec{\xi}(j)$ based on observations of the sequence $\{\vec{\xi}(j)+\vec{\eta}(j)\}$ at points $j \in \mathbb{Z}_{-} \backslash S$ can be calculated by formulas (4), (5), where $\mathbf{B}, \mathbf{R}, \mathbf{Q}$ are linear operators in the space $\ell_{2}$ defined by compound matrices constructed of coefficients $B(k, j), R(k, j), Q(k, j), k, j \in U,(U=S \cup\{0,1,2, \ldots\})$. For example, the matrix $B$ is of the form

$$
B=\left(\begin{array}{cc}
B_{s, s} & B_{s, n} \\
B_{n, s} & B_{n, n}
\end{array}\right),
$$

where its components are matrices constructed from the block-matrices

$$
\begin{array}{ll}
B_{s, n}(k, j)=B(k, j), & k=-M-N, \ldots,-M, \quad j=0,1,2, \ldots, \\
B_{n, s}(k, j)=B(k, j), & k=0,1,2, \ldots, \quad j=-M-N, \ldots,-M, \\
B_{n, n}(k, j)=B(k, j), & k, j=0,1,2, \ldots, \\
B_{s, s}(k, j)=B(k, j), & k=-M-N, \ldots,-M, \quad j=-M-N, \ldots,-M .
\end{array}
$$

Consider the problem of the mean-square optimal linear estimation of the functional $A \vec{\xi}$ which depends on the unknown values of the sequence $\{\vec{\xi}(j)\}$ from observations of the sequence $\{\vec{\xi}(j)+\vec{\eta}(j)\}$ at points $j \in \mathbb{Z}_{-} \backslash\{-s\}, Z^{S}=\{1,2, \ldots\} \backslash\{s\}$.

It follows from Theorem 1 that the following corollary holds true.

Corollary 2. Let $\vec{\xi}(j)$ and $\vec{\eta}(j)$ be uncorrelated multidimensional stationary sequences with spectral densities $F(\lambda)$ and $G(\lambda)$ which satisfy the minimality condition (1). The spectral characteristic $h\left(e^{i \lambda}\right)$ and the mean square error $\Delta(F, G)$ of the optimal linear estimate of the functional $A \vec{\xi}$ which depends on the unknown values of the sequence $\vec{\xi}(j)$ based on observations of the sequence $\vec{\xi}(j)+\vec{\eta}(j), j \in \mathbb{Z}_{-} \backslash\{-s\}$ can be calculated by formulas (4), (5), where $\mathbf{B}, \mathbf{R}, \mathbf{Q}$ are linear operators in the space $\ell_{2}$ defined by compound matrices constructed of coefficients $B(k, j), R(k, j), Q(k, j), k, j \in U,(U=S \cup\{0,1,2, \ldots\})$,

$$
B=\left(\begin{array}{cc}
B(-s,-s) & B_{-s, n} \\
B_{n,-s} & B_{n, n}
\end{array}\right),
$$

where elements in the last column and the last row are the matrices with the elements

$$
\begin{array}{ll}
B_{-s, n}(k, j)=B(k, j), & k=-s, \quad j=0,1,2, \ldots, \\
B_{n,-s}(k, j)=B(k, j), & k=0,1,2, \ldots, \quad j=-s, \\
B_{n, n}(k, j)=B(k, j), & k, j=0,1,2, \ldots
\end{array}
$$

Consider the problem of the mean-square optimal linear estimation of the functional

$$
A_{N} \vec{\xi}=\sum_{j \in Z^{S} \cap\{0, \ldots, N\}} \vec{a}(j)^{\top} \vec{\xi}(-j)
$$


which depends on the unknown values of the sequence $\vec{\xi}(j)$ from observations of the sequence $\vec{\xi}(j)+\vec{\eta}(j)$ at points $j \in \mathbb{Z}_{-} \backslash S$ where $S$ is defined in the introduction. The linear estimate of the functional $A_{N} \vec{\xi}$ has the representation

$$
\hat{A}_{N} \vec{\xi}=\int_{-\pi}^{\pi}\left(h_{N}\left(e^{i \lambda}\right)^{\top}\left(Z_{\tilde{\zeta}}(d \lambda)+Z_{\eta}(d \lambda)\right) .\right.
$$

Define the vector $\overrightarrow{\mathbf{a}}_{N}$ as follows: elements with indices from the set $U \cap(S \cup\{0, \ldots, N\})$ coincide with the elements of the vector $\overrightarrow{\mathbf{a}}$ with the same indices and elements with indices from the set $U \backslash(S \cup\{0, \ldots, N\})$ are zeros. $\mathbf{B}, \mathbf{R}, \mathbf{Q}$ are linear operators in the space $\ell_{2}$ defined in the Theorem 1.

The spectral characteristic $h_{N}\left(e^{i \lambda}\right)$ and the mean square error $\Delta\left(h_{N} ; F, G\right)$ of the optimal linear estimate of the functional $A_{N} \vec{\xi}$ can be calculated by formulas (6), (7)

$$
\begin{aligned}
&\left(h_{N}\left(e^{i \lambda}\right)\right)^{\top}=\left(A_{N}\left(e^{i \lambda}\right)\right)^{\top} F(\lambda)(F(\lambda)+G(\lambda))^{-1} \\
&-\left(\sum_{k \in U}\left(\mathbf{B}^{-1} \mathbf{R} \overrightarrow{\mathbf{a}}_{N}\right)(k) e^{i k \lambda}\right)^{\top}(F(\lambda)+G(\lambda))^{-1}, \\
& \Delta\left(h_{N} ; F, G\right)=\left\langle\mathbf{R} \overrightarrow{\mathbf{a}}_{N}, \mathbf{B}^{-1} \mathbf{R} \overrightarrow{\mathbf{a}}_{N}\right\rangle+\left\langle\mathbf{Q} \overrightarrow{\mathbf{a}}_{N}, \overrightarrow{\mathbf{a}}_{N}\right\rangle,
\end{aligned}
$$

where $A_{N}\left(e^{i \lambda}\right)=\sum_{j \in Z^{S} \cap\{0, \ldots, N\}} \vec{a}(j) e^{-i j \lambda}$.

The following corollary holds true.

Corollary 3. Let $\vec{\xi}(j)$ and $\vec{\eta}(j)$ be multidimensional uncorrelated stationary sequences with the spectral densities $F(\lambda)$ and $G(\lambda)$ which satisfy the minimality condition (1). The spectral characteristic $h_{N}\left(e^{i \lambda}\right)$ and the mean square error $\Delta\left(h_{N} ; F, G\right)$ of the optimal linear estimate of the functional $A_{N} \vec{\xi}$ which depends on the unknown values of the sequence $\vec{\xi}(j)$ from observation of the sequence $\{\vec{\xi}(j)+\vec{\eta}(j)\}$ at points of time $j \in \mathbb{Z}_{-} \backslash S$ can be calculated by formulas (6), (7).

\section{MinimaX-ROBUST METHOD OF FILTERING}

Theorem 1 and its corollaries can be applied to filtering of the functional in the cases when spectral densities of the sequences are exactly known. If complete information on the spectral densities is impossible but the class of admissible densities is given, it is reasonable to apply the minimax-robust method of filtering which consists in minimizing the value of the meansquare error for all spectral densities from the given class. For description of minimax method we propose the following definitions (see Moklyachuk and Masytka [29]).

Definition 1. For a given class of spectral densities $D=D_{F} \times D_{G}$ the spectral densities $F^{0}(\lambda) \in$ $D_{F}, G^{0}(\lambda) \in D_{G}$ are called least favorable in the class $D$ for the optimal linear filtering of the functional $A \vec{\xi}$ if the following relation holds true

$$
\Delta\left(F^{0}, G^{0}\right)=\Delta\left(h\left(F^{0}, G^{0}\right) ; F^{0}, G^{0}\right)=\max _{(F, G) \in D_{F} \times D_{G}} \Delta(h(F, G) ; F, G) .
$$


Definition 2. For a given class of spectral densities $D=D_{F} \times D_{G}$ the spectral characteristic $h^{0}\left(e^{i \lambda}\right)$ of the optimal linear estimate of the functional $A \vec{\xi}$ is called minimax-robust if there are satisfied conditions

$$
\begin{gathered}
h^{0}\left(e^{i \lambda}\right) \in H_{D}=\bigcap_{(F, G) \in D_{F} \times D_{G}} L_{2}^{s}(F+G), \\
\min _{h \in H_{D}} \max _{(F, G) \in D} \Delta(h ; F, G)=\max _{(F, G) \in D} \Delta\left(h^{0} ; F, G\right) .
\end{gathered}
$$

From the introduced definitions and formulas derived above we can obtain the following statement.

Lemma 1. Spectral densities $F^{0}(\lambda) \in D_{F}, G^{0}(\lambda) \in D_{G}$ satisfying the minimality condition (1) are the least favorable in the class $D=D_{F} \times D_{G}$ for the optimal linear filtering of the functional $A \vec{\xi}$ if operators $B^{0}, R^{0}, Q^{0}$ determined by the Fourier coefficients of the functions

$$
\left(F^{0}(\lambda)+G^{0}(\lambda)\right)^{-1}, F^{0}(\lambda)\left(F^{0}(\lambda)+G^{0}(\lambda)\right)^{-1}, F^{0}(\lambda)\left(F^{0}(\lambda)+G^{0}(\lambda)\right)^{-1} G^{0}(\lambda)
$$

determine a solution to the constrain optimization problem

$$
\max _{(F, G) \in D_{F} \times D_{G}}\left\langle\mathbf{R} \overrightarrow{\mathbf{a}}, \mathbf{B}^{-1} \mathbf{R} \overrightarrow{\mathbf{a}}\right\rangle+\langle\mathbf{Q} \overrightarrow{\mathbf{a}}, \overrightarrow{\mathbf{a}}\rangle=\left\langle\mathbf{R}^{0} \overrightarrow{\mathbf{a}},\left(\mathbf{B}^{0}\right)^{-1} \mathbf{R}^{0} \overrightarrow{\mathbf{a}}\right\rangle+\left\langle\mathbf{Q}^{0} \overrightarrow{\mathbf{a}}, \overrightarrow{\mathbf{a}}\right\rangle .
$$

The minimax spectral characteristic $h^{0}=h\left(F^{0}, G^{0}\right)$ is determined by the formula (4) if $h\left(F^{0}, G^{0}\right) \in H_{D}$.

The least favorable spectral densities $F^{0}(\lambda), G^{0}(\lambda)$ and the minimax spectral characteristic $h^{0}=h\left(F^{0}, G^{0}\right)$ form a saddle point of the function $\Delta(h ; F, G)$ on the set $H_{D} \times D$. The saddle point inequalities

$$
\begin{gathered}
\Delta\left(h ; F^{0}, G^{0}\right) \geq \Delta\left(h^{0} ; F^{0}, G^{0}\right) \geq \Delta\left(h^{0} ; F, G\right) \\
\forall h \in H_{D}, \forall \quad F \in D_{F}, \forall \quad G \in D_{G}
\end{gathered}
$$

hold true if $h^{0}=h\left(F^{0}, G^{0}\right)$ and $h\left(F^{0}, G^{0}\right) \in H_{D}$, where $\left(F^{0}, G^{0}\right)$ is a solution to the constrained optimization problem

$$
\sup _{(F, G) \in D_{F} \times D_{G}} \Delta\left(h\left(F^{0}, G^{0}\right) ; F, G\right)=\Delta\left(h\left(F^{0}, G^{0}\right) ; F^{0}, G^{0}\right),
$$

where the functional $\Delta\left(h\left(F^{0}, G^{0}\right) ; F, G\right)$ is calculated by the formula

$$
\begin{aligned}
& \Delta\left(h\left(F^{0}, G^{0}\right) ; F, G\right)=\frac{1}{2 \pi} \int_{-\pi}^{\pi}\left(r_{G}^{0}(\lambda)\right)^{\top} F(\lambda) \overline{r_{G}^{0}(\lambda)} d \lambda+\frac{1}{2 \pi} \int_{-\pi}^{\pi}\left(r_{F}^{0}(\lambda)\right)^{\top} G(\lambda) \overline{r_{F}^{0}(\lambda)} d \lambda, \\
& \left(r_{F}^{0}(\lambda)\right)^{\top}=\left(\left(A\left(e^{i \lambda}\right)\right)^{\top} F^{0}(\lambda)-\left(\sum_{k \in U}\left(\left(\mathbf{B}^{0}\right)^{-1} \mathbf{R}^{0} \overrightarrow{\mathbf{a}}\right)(k) e^{i k \lambda}\right)^{\top}\right)\left(F^{0}(\lambda)+G^{0}(\lambda)\right)^{-1}, \\
& \left(r_{G}^{0}(\lambda)\right)^{\top}=\left(\left(A\left(e^{i \lambda}\right)\right)^{\top} G^{0}(\lambda)+\left(\sum_{k \in U}\left(\left(\mathbf{B}^{0}\right)^{-1} \mathbf{R}^{0} \overrightarrow{\mathbf{a}}\right)(k) e^{i k \lambda}\right)^{\top}\right)\left(F^{0}(\lambda)+G^{0}(\lambda)\right)^{-1} .
\end{aligned}
$$


The constrained optimization problem (9) is equivalent to the unconstrained optimization problem (see, for example, Pshenichnyj [36]):

$$
\Delta_{D}(F, G)=-\Delta\left(h\left(F^{0}, G^{0}\right) ; F, G\right)+\delta\left((F, G) \mid D_{F} \times D_{G}\right) \rightarrow \text { inf, }
$$

where $\delta\left((F, G) \mid D_{F} \times D_{G}\right)$ is the indicator function of the set $D=D_{F} \times D_{G}$. Solution of the problem (10) is characterized by the condition $0 \in \partial \Delta_{D}\left(F^{0}, G^{0}\right)$, where $\partial \Delta_{D}\left(F^{0}, G^{0}\right)$ is the subdifferential of the convex functional $\Delta_{D}(F, G)$ at point $\left(F^{0}, G^{0}\right)$. This condition makes it possible to find the least favourable spectral densities in some special classes of spectral densities $D$ (see books by Ioffe and Tihomirov [13], Pshenichnyj [36], Rockafellar [37]).

Note, that the form of the functional $\Delta\left(h^{0} ; F, G\right)$ is convenient for application the Lagrange method of indefinite multipliers for finding solution to the problem (10). Making use the method of Lagrange multipliers and the form of subdifferentials of the indicator functions we describe relations that determine least favourable spectral densities in some special classes of spectral densities (see books by Moklyachuk [24, 25], Moklyachuk and Masyutka [29] for additional details).

\section{Least FaVORABLE SPECTRAL DENSITIES IN THE CLASS $D=D_{0} \times D_{2 \delta}$}

Consider the problem of filtering of the functional $A \vec{\xi}$ in the case where spectral densities $F(\lambda), G(\lambda)$ belong to the set of admissible spectral densities $D_{0} \times D_{2 \delta}$, where

$$
\begin{gathered}
D_{0}^{1}=\left\{F(\lambda) \mid \frac{1}{2 \pi} \int_{-\pi}^{\pi} \operatorname{Tr} F(\lambda) d \lambda=p\right\}, \\
D_{2 \delta}^{1}=\left\{\left.G(\lambda)\left|\frac{1}{2 \pi} \int_{-\pi}^{\pi}\right| \operatorname{Tr}\left(G(\lambda)-G_{1}(\lambda)\right)\right|^{2} d \lambda \leq \delta\right\} ; \\
D_{0}^{2}=\left\{F(\lambda) \mid \frac{1}{2 \pi} \int_{-\pi}^{\pi} f_{k k}(\lambda) d \lambda=p_{k}, k=\overline{1, T}\right\}, \\
D_{2 \delta}^{2}=\left\{G(\lambda)\left|\frac{1}{2 \pi} \int_{-\pi}^{\pi}\right| g_{k k}(\lambda)-\left.g_{k k}^{1}(\lambda)\right|^{2} d \lambda \leq \delta_{k}, k=\overline{1, T}\right\} ; \\
D_{2 \delta}^{3}=\left\{F(\lambda) \mid \frac{1}{2 \pi} \int_{-\pi}^{\pi}\left\langle B_{1}, F(\lambda)\right\rangle d \lambda=p\right\}, \\
D_{2 \delta}^{3}=\left\{\left.G(\lambda)\left|\frac{1}{2 \pi} \int_{-\pi}^{\pi}\right|\left\langle B_{2}, G(\lambda)-G_{1}(\lambda)\right\rangle\right|^{2} d \lambda \leq \delta\right\} ; \\
D_{0}^{4}=\left\{F(\lambda) \mid \frac{1}{2 \pi} \int_{-\pi}^{\pi} F(\lambda) d \lambda=P\right\},
\end{gathered}
$$

Here the spectral density $\left.G_{1}(\lambda)\right)$ is known and fixed, $p, \delta, p_{k}, \delta_{k}, k=\overline{1, T}, \delta_{i}^{j}, i, j=\overline{1, T}$, are fixed numbers, $P, B_{1}, B_{2}$ are fixed positive definite Hermitian matrices.

The classes $D_{0}^{k}, k=\overline{1,4}$ describe densities with the moment restrictions while the classes $D_{2 \delta^{\prime}}^{k} k=\overline{1,4}$ describe the " $\delta$-neighborhood" models in the space $L_{2}$ of a fixed bounded spectral density $G_{1}(\lambda)$. 
From the condition $0 \in \partial \Delta_{D}\left(F^{0}, G^{0}\right)$ we find the following equations which determine the least favourable spectral densities for these given sets of admissible spectral densities.

For the first pair $D_{0}^{1} \times D_{2 \delta}^{1}$ we have equations

$$
\begin{gathered}
\left(r_{G}^{0}(\lambda)\right)^{*}\left(r_{G}^{0}(\lambda)\right)^{\top}=\alpha^{2}\left(F^{0}(\lambda)+G^{0}(\lambda)\right)^{2}, \\
\left(r_{F}^{0}(\lambda)\right)^{*}\left(r_{F}^{0}(\lambda)\right)^{\top}=\beta^{2} \operatorname{Tr}\left(G^{0}(\lambda)-G_{1}(\lambda)\right)\left(F^{0}(\lambda)+G^{0}(\lambda)\right)^{2}, \\
\frac{1}{2 \pi} \int_{-\pi}^{\pi}\left|\operatorname{Tr}\left(G(\lambda)-G_{1}(\lambda)\right)\right|^{2} d \lambda=\delta,
\end{gathered}
$$

where $\alpha^{2}, \beta^{2}$ are Lagrange multipliers.

For the second pair $D_{0}^{2} \times D_{2 \delta}^{2}$ we have equations

$$
\begin{gathered}
\left(r_{G}^{0}(\lambda)\right)^{*}\left(r_{G}^{0}(\lambda)\right)^{\top}=\left(F^{0}(\lambda)+G^{0}(\lambda)\right)\left\{\alpha_{k}^{2} \delta_{k l}\right\}_{k, l=1}^{T}\left(F^{0}(\lambda)+G^{0}(\lambda)\right), \\
\left(r_{F}^{0}(\lambda)\right)^{*}\left(r_{F}^{0}(\lambda)\right)^{\top}=\left(F^{0}(\lambda)+G^{0}(\lambda)\right)\left\{\beta_{k}^{2}\left(g_{k k}^{0}(\lambda)-g_{k k}^{1}(\lambda)\right) \delta_{k l}\right\}_{k, l=1}^{T}\left(F^{0}(\lambda)+G^{0}(\lambda)\right), \\
\frac{1}{2 \pi} \int_{-\pi}^{\pi}\left|g_{k k}(\lambda)-g_{k k}^{1}(\lambda)\right|^{2} d \lambda=\delta_{k}, k=\overline{1, T,}
\end{gathered}
$$

where $\alpha_{k}^{2}, \beta_{k}^{2}$ are Lagrange multipliers.

For the third pair $D_{0}^{3} \times D_{2 \delta}^{3}$ we have equations

$$
\begin{gathered}
\left(r_{G}^{0}(\lambda)\right)^{*}\left(r_{G}^{0}(\lambda)\right)^{\top}=\alpha^{2}\left(F^{0}(\lambda)+G^{0}(\lambda)\right) B_{1}^{\top}\left(F^{0}(\lambda)+G^{0}(\lambda)\right), \\
\left(r_{F}^{0}(\lambda)\right)^{*}\left(r_{F}^{0}(\lambda)\right)^{\top}=\beta^{2}\left\langle B_{2}, G^{0}(\lambda)-G_{1}(\lambda)\right\rangle\left(F^{0}(\lambda)+G^{0}(\lambda)\right)^{2}, \\
\frac{1}{2 \pi} \int_{-\pi}^{\pi}\left|\left\langle B_{2}, G(\lambda)-G_{1}(\lambda)\right\rangle\right|^{2} d \lambda=\delta,
\end{gathered}
$$

where $\alpha^{2}, \beta^{2}$ are Lagrange multipliers.

For the fourth pair $D_{0}^{4} \times D_{2 \delta}^{4}$ we have equations

$$
\begin{gathered}
\left(r_{G}^{0}(\lambda)\right)^{*}\left(r_{G}^{0}(\lambda)\right)^{\top}=\left(F^{0}(\lambda)+G^{0}(\lambda)\right) \vec{\alpha} \cdot \vec{\alpha}^{*}\left(F^{0}(\lambda)+G^{0}(\lambda)\right), \\
\left(r_{F}^{0}(\lambda)\right)^{*}\left(r_{F}^{0}(\lambda)\right)^{\top}=\left(F^{0}(\lambda)+G^{0}(\lambda)\right)\left\{\beta_{i j}\left(g_{i j}^{0}(\lambda)-g_{i j}^{1}(\lambda)\right)\right\}_{i, j=1}^{T}\left(F^{0}(\lambda)+G^{0}(\lambda)\right), \\
\frac{1}{2 \pi} \int_{-\pi}^{\pi}\left|g_{i j}(\lambda)-g_{i j}^{1}(\lambda)\right|^{2} d \lambda=\delta_{i}^{j}, i, j=\overline{1, T},
\end{gathered}
$$

where $\vec{\alpha}, \beta_{i j}$ are Lagrange multipliers.

The following theorem and corollaries hold true.

Theorem 2. The least favorable spectral densities $F^{0}(\lambda), G^{0}(\lambda)$ in the classes $D_{0}^{k} \times D_{2 \delta^{\prime}}^{k} k=\overline{1,4}$, for the optimal linear filtering of the functional $A \vec{\xi}$ are determined by relations (11) - (13) for the first pair $D_{0}^{1} \times D_{2 \delta}^{1}$ of sets of admissible spectral densities; (14) - (16) for the second pair $D_{0}^{2} \times D_{2 \delta}^{2}$ of sets of admissible spectral densities; (17) - (19) for the third pair $D_{0}^{3} \times D_{2 \delta}^{3}$ of sets of admissible spectral densities; (20) - (22) for the fourth pair $D_{0}^{4} \times D_{2 \delta}^{4}$ of sets of admissible spectral densities; the minimality condition (1), the constrained optimization problem (8) and restrictions on densities from the corresponding classes $D_{0} \times D_{2 \delta}$. The minimax-robust spectral characteristic of the optimal estimate of the functional $A \vec{\xi}$ is determined by the formula (4). 
Corollary 4. Assume that the spectral density $G(\lambda)$ is known. Let the function $F^{0}(\lambda)+G(\lambda)$ satisfies the minimality condition (1). The spectral density $F^{0}(\lambda)$ is the least favorable in the classes $D_{0}^{k}, k=\overline{1,4}$, for the optimal linear filtering of the functional $A \vec{\xi}$ if it satisfies relations (11), (14), (17), (20), respectively, and the pair $\left(F^{0}(\lambda), G(\lambda)\right)$ is a solution of the optimization problem (8). The minimax-robust spectral characteristic of the optimal estimate of the functional $A \vec{\xi}$ is determined by formula (4).

Corollary 5. Assume that the spectral density $F(\lambda)$ is known. Let the function $F(\lambda)+G^{0}(\lambda)$ satisfies the minimality condition (1). The spectral density $G^{0}(\lambda)$ is the least favorable in the classes $D_{2 \delta}^{k}, k=\overline{1,4}$, for the optimal linear filtering of the functional $A \vec{\xi}$ if it satisfies relations (12) - (13), (15) - (16), (18) - (19), (21) - (22), respectively, and the pair $\left(F(\lambda), G^{0}(\lambda)\right)$ is a solution of the optimization problem (8). The minimax-robust spectral characteristic of the optimal estimate of the functional $A \vec{\xi}$ is determined by formula (4).

\section{LeAst FaVOrable SPECTRAL DENSITIES IN THE CLASS $D=D_{1 \delta} \times D_{V}^{U}$}

Consider the problem of filtering of the functional $A \vec{\xi}$ in the case where spectral densities $F(\lambda), G(\lambda)$ belong to the set of admissible spectral densities $D_{1 \delta} \times D_{V}^{U}$, where

$$
\begin{gathered}
D_{1 \delta}^{1}=\left\{F(\lambda)\left|\frac{1}{2 \pi} \int_{-\pi}^{\pi}\right| \operatorname{Tr}\left(F(\lambda)-F_{1}(\lambda)\right) \mid d \lambda \leq \delta\right\}, \\
D_{V}^{U^{1}}=\left\{G(\lambda) \mid \operatorname{Tr} V(\lambda) \leq \operatorname{Tr} G(\lambda) \leq \operatorname{Tr} U(\lambda), \frac{1}{2 \pi} \int_{-\pi}^{\pi} \operatorname{Tr} G(\lambda) d \lambda=q\right\}, \\
D_{1 \delta}^{2}=\left\{F(\lambda)\left|\frac{1}{2 \pi} \int_{-\pi}^{\pi}\right| f_{k k}(\lambda)-f_{k k}^{1}(\lambda) \mid d \lambda \leq \delta_{k}, k=\overline{1, T}\right\}, \\
D_{V}^{U^{2}}=\left\{G(\lambda) \mid v_{k k}(\lambda) \leq g_{k k}(\lambda) \leq u_{k k}(\lambda), \frac{1}{2 \pi} \int_{-\pi}^{\pi} g_{k k}(\lambda) d \lambda=q_{k}, k=\overline{1, T}\right\}, \\
D_{V}^{U^{3}}=\left\{G(\lambda) \mid\left\langle B_{2}, V(\lambda)\right\rangle \leq\left\langle B_{2}, G(\lambda)\right\rangle \leq\left\langle B_{2}, U(\lambda)\right\rangle, \frac{1}{2 \pi} \int_{-\pi}^{\pi}\left\langle B_{2}, G(\lambda)\right\rangle d \lambda=q\right\}, \\
D_{1 \delta}^{4}=\left\{F(\lambda)\left|\frac{1}{2 \pi} \int_{-\pi}^{\pi}\right| f_{i j}(\lambda)-f_{i j}^{1}(\lambda) \mid d \lambda \leq \delta_{i}^{j}, i, j=\overline{1, T}\right\}, \\
D_{V}^{U^{4}}=\left\{G(\lambda) \mid V(\lambda) \leq G(\lambda) \leq U(\lambda), \frac{1}{2 \pi} \int_{-\pi}^{\pi} G(\lambda) d \lambda=Q\right\} .
\end{gathered}
$$

Here the spectral densities $F_{1}(\lambda), V(\lambda), U(\lambda)$ are known and fixed, $\delta, q, \delta_{k}, q_{k}, k=\overline{1, T}, \delta_{i}^{j}, i, j=$ $\overline{1, T}$, are fixed numbers, $Q, B_{1}, B_{2}$ are fixed positive definite Hermitian matrices.

The classes $D_{V}^{U^{k}}, k=\overline{1,4}$ describe the "strip" models of spectral densities while the classes $D_{1 \delta}^{k}, k=\overline{1,4}$ describe " $\delta$-neighborhood" model in the space $L_{1}$ of a fixed bounded spectral density $F_{1}(\lambda)$.

From the condition $0 \in \partial \Delta_{D}\left(F^{0}, G^{0}\right)$ we find the following equations which determine the least favourable spectral densities for these sets of admissible spectral densities. 
For the first pair $D_{1 \delta}^{1} \times D_{V}^{U^{1}}$ we have equations

$$
\begin{gathered}
\left(r_{G}^{0}(\lambda)\right)^{*}\left(r_{G}^{0}(\lambda)\right)^{\top}=\alpha^{2} \gamma(\lambda)\left(F^{0}(\lambda)+G^{0}(\lambda)\right)^{2}, \\
\frac{1}{2 \pi} \int_{-\pi}^{\pi}\left|\operatorname{Tr}\left(F^{0}(\lambda)-F_{1}(\lambda)\right)\right| d \lambda=\delta, \\
\left(r_{F}^{0}(\lambda)\right)^{*}\left(r_{F}^{0}(\lambda)\right)^{\top}=\left(\beta^{2}+\gamma_{1}(\lambda)+\gamma_{2}(\lambda)\right)\left(F^{0}(\lambda)+G^{0}(\lambda)\right)^{2},
\end{gathered}
$$

where $\alpha^{2}, \beta^{2}$ are Lagrange multipliers, $|\gamma(\lambda)| \leq 1$ and

$$
\gamma(\lambda)=\operatorname{sign}\left(\operatorname{Tr}\left(F^{0}(\lambda)-F_{1}(\lambda)\right)\right): \operatorname{Tr}\left(F^{0}(\lambda)-F_{1}(\lambda)\right) \neq 0,
$$

$\gamma_{1}(\lambda) \leq 0$ and $\gamma_{1}(\lambda)=0$ if $\operatorname{Tr} G^{0}(\lambda)>\operatorname{Tr} V(\lambda), \gamma_{2}(\lambda) \geq 0$ and $\gamma_{2}(\lambda)=0$ if $\operatorname{Tr} G^{0}(\lambda)<$ $\operatorname{Tr} U(\lambda)$.

For the second pair $D_{1 \delta}^{2} \times D_{V}^{U^{2}}$ we have equations

$$
\begin{gathered}
\left(r_{G}^{0}(\lambda)\right)^{*}\left(r_{G}^{0}(\lambda)\right)^{\top}=\left(F^{0}(\lambda)+G^{0}(\lambda)\right)\left\{\alpha_{k}^{2} \gamma_{k}(\lambda) \delta_{k l}\right\}_{k, l=1}^{T}\left(F^{0}(\lambda)+G^{0}(\lambda)\right), \\
\frac{1}{2 \pi} \int_{-\pi}^{\pi}\left|f_{k k}^{0}(\lambda)-f_{k k}^{1}(\lambda)\right| d \lambda=\delta_{k}, k=\overline{1, T}, \\
\left(r_{F}^{0}(\lambda)\right)^{*}\left(r_{F}^{0}(\lambda)\right)^{\top}=\left(F^{0}(\lambda)+G^{0}(\lambda)\right)\left\{\left(\beta_{k}^{2}+\gamma_{1 k}(\lambda)+\gamma_{2 k}(\lambda)\right) \delta_{k l}\right\}_{k, l=1}^{T}\left(F^{0}(\lambda)+G^{0}(\lambda)\right),
\end{gathered}
$$

where $\alpha_{k}^{2}, \beta_{k}^{2}$ are Lagrange multipliers, $\left|\gamma_{k}(\lambda)\right| \leq 1$ and

$$
\gamma_{k}(\lambda)=\operatorname{sign}\left(f_{k k}^{0}(\lambda)-f_{k k}^{1}(\lambda)\right): f_{k k}^{0}(\lambda)-f_{k k}^{1}(\lambda) \neq 0, k=\overline{1, T}
$$

$\gamma_{1 k}(\lambda) \leq 0$ and $\gamma_{1 k}(\lambda)=0$ if $g_{k k}^{0}(\lambda)>v_{k k}(\lambda), \gamma_{2 k}(\lambda) \geq 0$ and $\gamma_{2 k}(\lambda)=0$ if $g_{k k}^{0}(\lambda)<u_{k k}(\lambda)$.

For the third pair $D_{1 \delta}^{3} \times D_{V}^{U^{3}}$ we have equations

$$
\begin{gathered}
\left(r_{G}^{0}(\lambda)\right)^{*}\left(r_{G}^{0}(\lambda)\right)^{\top}=\alpha^{2} \gamma^{\prime}(\lambda)\left(F^{0}(\lambda)+G^{0}(\lambda)\right) B_{1}^{\top}\left(F^{0}(\lambda)+G^{0}(\lambda)\right), \\
\frac{1}{2 \pi} \int_{-\pi}^{\pi}\left|\left\langle B_{1}, F^{0}(\lambda)-F_{1}(\lambda)\right\rangle\right| d \lambda=\delta, \\
\left(r_{F}^{0}(\lambda)\right)^{*}\left(r_{F}^{0}(\lambda)\right)^{\top}=\left(\beta^{2}+\gamma_{1}^{\prime}(\lambda)+\gamma_{2}^{\prime}(\lambda)\right)\left(F^{0}(\lambda)+G^{0}(\lambda)\right) B_{2}^{\top}\left(F^{0}(\lambda)+G^{0}(\lambda)\right),
\end{gathered}
$$

where $\alpha^{2}, \beta^{2}$ are Lagrange multipliers, $\left|\gamma^{\prime}(\lambda)\right| \leq 1$ and

$$
\gamma^{\prime}(\lambda)=\operatorname{sign}\left\langle B_{1}, F^{0}(\lambda)-F_{1}(\lambda)\right\rangle:\left\langle B_{1}, F^{0}(\lambda)-F_{1}(\lambda)\right\rangle \neq 0,
$$

$\gamma_{1}^{\prime}(\lambda) \leq 0$ and $\gamma_{1}^{\prime}(\lambda)=0$ if $\left\langle B_{2}, G^{0}(\lambda\rangle>\left\langle B_{2}, V(\lambda)\right\rangle, \gamma_{2}^{\prime}(\lambda) \geq 0\right.$ and $\gamma_{2}^{\prime}(\lambda)=0$ if $\left\langle B_{2}, G^{0}(\lambda\rangle<\left\langle B_{2}, U(\lambda)\right\rangle\right.$.

For the fourth pair $D_{1 \delta}^{4} \times D_{V}^{U^{4}}$ we have equations

$$
\begin{gathered}
\left.\left(r_{G}^{0}(\lambda)\right)^{*}\left(r_{G}^{0}(\lambda)\right)^{\top}=\left(F^{0}(\lambda)+G^{0}(\lambda)\right)\left\{\alpha_{i j} \gamma_{i j}(\lambda)\right)\right\}_{i, j=1}^{T}\left(F^{0}(\lambda)+G^{0}(\lambda)\right), \\
\frac{1}{2 \pi} \int_{-\pi}^{\pi}\left|f_{i j}^{0}(\lambda)-f_{i j}^{1}(\lambda)\right| d \lambda=\delta_{i}^{j}, i, j=\overline{1, T}, \\
\left(r_{F}^{0}(\lambda)\right)^{*}\left(r_{F}^{0}(\lambda)\right)^{\top}=\left(F^{0}(\lambda)+G^{0}(\lambda)\right)\left(\vec{\beta} \cdot \vec{\beta}^{*}+\Gamma_{1}(\lambda)+\Gamma_{2}(\lambda)\right)\left(F^{0}(\lambda)+G^{0}(\lambda)\right)
\end{gathered}
$$

where $\vec{\beta}, \alpha_{i j}$ are Lagrange multipliers, $\left|\gamma_{i j}(\lambda)\right| \leq 1$ and

$$
\gamma_{i j}(\lambda)=\frac{f_{i j}^{0}(\lambda)-f_{i j}^{1}(\lambda)}{\left|f_{i j}^{0}(\lambda)-f_{i j}^{1}(\lambda)\right|}: f_{i j}^{0}(\lambda)-f_{i j}^{1}(\lambda) \neq 0, i, j=\overline{1, T},
$$

$\Gamma_{1}(\lambda) \leq 0$ and $\Gamma_{1}(\lambda)=0$ if $G^{0}(\lambda)>V(\lambda), \Gamma_{2}(\lambda) \geq 0$ and $\Gamma_{2}(\lambda)=0$ if $G^{0}(\lambda)<U(\lambda)$. 
The following theorem and corollaries hold true.

Theorem 3. The least favorable spectral densities $F^{0}(\lambda), G^{0}(\lambda)$ in the classes $D_{1 \delta}^{k} \times D_{V}^{U^{k}}, k=$ $\overline{1,4}$, for the optimal linear filtering of the functional $A \vec{\xi}$ are determined by relations (23) - (25) for the first pair $D_{1 \delta}^{4} \times D_{V}^{U^{1}}$ of sets of admissible spectral densities; (26) - (28) for the second pair $D_{1 \delta}^{4} \times D_{V}^{U^{2}}$ of sets of admissible spectral densities; (29) - (31) for the third pair $D_{1 \delta}^{4} \times D_{V}^{U^{3}}$ of sets of admissible spectral densities; (32) - (34) for the fourth pair $D_{1 \delta}^{4} \times D_{V}^{U^{4}}$ of sets of admissible spectral densities; the minimality condition (1), the constrained optimization problem (8) and restrictions on densities from the corresponding classes $D_{1 \delta} \times D_{V}^{U}$. The minimax-robust spectral characteristic of the optimal estimate of the functional $A \vec{\xi}$ is determined by the formula (4).

Corollary 6. Assume that the spectral density $G(\lambda)$ is known. Let the function $F^{0}(\lambda)+G(\lambda)$ satisfies the minimality condition (1). The spectral density $F^{0}(\lambda)$ is the least favorable in the classes $D_{1 \delta}^{k}, k=\overline{1,4}$, for the optimal linear filtering of the functional $A \vec{\xi}$ if it satisfies relations (23) - (24), (26) - (27), (29) - (30), (32) - (33), respectively, and the pair $\left(F^{0}(\lambda), G(\lambda)\right)$ is a solution of the optimization problem (8). The minimax-robust spectral characteristic of the optimal estimate of the functional $A \vec{\xi}$ is determined by formula (4).

Corollary 7. Assume that the spectral density $F(\lambda)$ is known. Let the function $F(\lambda)+G^{0}(\lambda)$ satisfies the minimality condition (1). The spectral density $G^{0}(\lambda)$ is the least favorable in the classes $D_{V}^{U^{k}}, k=\overline{1,4}$, for the optimal linear filtering of the functional $A \vec{\xi}$ if it satisfies relations (25), (28), (31), (34), respectively, and the pair $\left(F(\lambda), G^{0}(\lambda)\right)$ is a solution of the optimization problem (8). The minimax-robust spectral characteristic of the optimal estimate of the functional $A \vec{\xi}$ is determined by formula (4).

\section{CONCLUSIONS}

In the article we propose methods of the mean-square optimal linear filtering of functionals which depend on the unknown values of a multidimensional stationary stochastic sequence. Estimates are based on observations of the sequence with an additive stationary noise sequence. We develop methods of finding the optimal estimates of the functionals in the case of missing observations. The problem is investigated in the case of spectral certainty, where the spectral densities of the sequences are exactly known. In this case we propose an approach based on the Hilbert space projection method. We derive formulas for calculating the spectral characteristic and the mean-square error of the optimal estimate of the functionals. In the case of spectral uncertainty, where the spectral densities of the stationary sequences are not exactly known while some special sets of admissible spectral densities are given, we apply the minimax-robust estimation method of estimation. This method allows us to find estimates that minimize the maximum values of the mean-square errors of the estimates for all spectral density matrices from a given class of admissible spectral density matrices. We derive formulas that determine the least favorable spectral densities and the minimax spectral characteristics for some special sets of admissible spectral densities.

These least favourable spectral density matrices are solutions of the optimization problem $\Delta_{D}(F, G)=-\Delta\left(h\left(F^{0}, G^{0}\right) ; F, G\right)+\delta\left((F, G) \mid D_{F} \times D_{G}\right) \rightarrow$ inf, which is characterized by the condition $0 \in \partial \Delta_{D}\left(F^{0}, G^{0}\right)$, where $\partial \Delta_{D}\left(F^{0}, G^{0}\right)$ is the subdifferential of the convex functional 
$\Delta_{D}(F, G)$ at point $\left(F^{0}, G^{0}\right)$. The form of the functional $\Delta\left(h\left(F^{0}, G^{0}\right) ; F, G\right)$ is convenient for application of the Lagrange method of indefinite multipliers for finding solution to the optimization problem. The complexity of solution of the problem is determined by the complexity of calculating of subdifferentials of the indicator functions $\delta\left((f, g) \mid D_{f} \times D_{g}\right)$ of sets $D_{f} \times D_{g}$. Making use of the method of Lagrange multipliers and the form of subdifferentials of the indicator functions we describe relations that determine the least favourable spectral densities in some special classes of spectral densities. These are: classes $D_{0}$ of densities with the moment restrictions, classes $D_{1 \delta}$ which describe the " $\delta$-neighborhood" models in the space $L_{1}$ of a fixed bounded spectral density, classes $D_{2 \delta}$ which describe the " $\delta$-neighborhood" models in the space $L_{2}$ of a fixed bounded spectral density, classes $D_{V}^{U}$ which describe the "strip" models of spectral densities.

\section{REFERENCES}

[1] Bondon P. Prediction with incomplete past of a stationary process. Stochastic Process. Appl. 2002, 98, 67-76. doi:10.1016/S0304-4149(01)00116-8

[2] Bondon P. Influence of missing values on the prediction of a stationary time series. J. Time Ser. Anal. 2005,26 (4), 519-525. doi:10.1111/j.1467-9892.2005.00433.x

[3] Box G.E.P., Jenkins G.M., Reinsel G.C., Ljung G.M. Time series analysis. Forecasting and control. 5th ed. Wiley, 2016.

[4] Brockwell P.J., Davis R.A. Time series: Theory and methods. 2nd ed. Springer, New York, 1998.

[5] Cheng R., Miamee A.G., Pourahmadi M. Some extremal problems in $L^{p}(w)$. Proc. Amer. Math. Soc. 1998, 126, 2333-2340. doi:10.1090/S0002-9939-98-04275-0

[6] Cheng R., Pourahmadi M. Prediction with incomplete past and interpolation of missing values. Stat. Probab. Lett. 1996, 33, 341-346. doi:10.1016/S0167-7152(96)00146-0

[7] Franke J. On the robust prediction and interpolation of time series in the presence of correlated noise. J. Time Ser. Anal. 1984, 5 (4), 227-244. doi:10.1111/j.1467-9892.1984.tb00389.x

[8] Franke J. Minimax robust prediction of discrete time series. Z. Wahrscheinlichkeitstheor. Verw. Geb. 1985, 68, 337-364. doi:10.1007/BF00532645

[9] Franke J., Poor H.V. Minimax-robust filtering and finite-length robust predictors. Robust and Nonlinear Time Series Analysis. Lecture Notes in Statistics, Springer-Verlag, 1984, 26, 87-126.

[10] Gikhman I.I., Skorokhod A.V. The theory of stochastic processes. I. Springer, Berlin, 2004.

[11] Grenander U. A prediction problem in game theory. Ark. Mat. 1957, 26, 371-379.

[12] Hannan E.J. Multiple time series. Wiley, New York, 1970. doi:10.1002/9780470316429

[13] Ioffe A.D., Tihomirov V.M. Theory of extremal problems. North-Holland, Amsterdam, New York, Oxford, 1979.

[14] Karhunen K. Über lineare Methoden in der Wahrscheinlichkeitsrechnung. Ann. Acad. Sci. Fenn., Ser. A I, 1947, 37, $1-79$.

[15] Kasahara Y., Pourahmadi M., Inoue A. Duals of random vectors and processes with applications to prediction problems with missing values. Stat. Probab. Lett. 2009, 79 (14), 1637-1646. doi:10.1016/j.spl.2009.04.005

[16] Kassam S.A., Poor H.V. Robust techniques for signal processing: A survey. Proc. IEEE 1985, 73 (3), $433-481$. doi:10.1109/PROC.1985.13167

[17] Kolmogorov A.N. In: Shiryayev A. N. (Ed.) Selected works by A. N. Kolmogorov. Vol. II: Probability theory and mathematical statistics, Kluwer, Dordrecht etc., 1992. 
[18] Luz M. M., Moklyachuk M.P. Minimax-robust filtering problem for stochastic sequences with stationary increments. Theory Probab. Math. Statist. 2014, 89, 127-142. doi:10.1090/S0094-9000-2015-00940-6 (translation of Teor. Imovir. ta Matem. Statist. 2013, 89, 115-129. (in English))

[19] Luz M. M., Moklyachuk M. P. Minimax-robust filtering problem for stochastic sequences with stationary increments and cointegrated sequences. Stat., Optim. Inf. Comput. 2014, 2 (3), 176-199. doi:10.19139/soic.v2i3.56

[20] Luz M. M., Moklyachuk M. P. Minimax-robust filtering problem for stochastic sequences with stationary increments and cointegrated sequences. Cogent Mathematics 2016, 3, 1-21. doi:10.1080/23311835.2016.1167811

[21] Luz M.M., Moklyachuk M.P. Filtering problem for functionals of stationary sequences. Stat., Optim. Inf. Comput. 2016, 4 (1), 68-83. doi:10.19139/soic.v4i1.172

[22] Luz M. M., Moklyachuk M. P. Estimates of functionals from processes with stationary increments and cointegrated sequences. NVP "Interservis", Kyiv, 2016. (in Ukrainian)

[23] Moklyachuk M.P. On a filtering problem for vector-valued sequences. Theory Probab. Math. Statist. 1992, 47, 107-118. (translation of Teor. Imovir. ta Matem. Statist. 1992, 47, 104-117. (in Ukrainian))

[24] Moklyachuk M.P. Nonsmooth analysis and optimization. Kyiv University, Kyiv, 2008. (in Ukrainian)

[25] Moklyachuk M.P. Robust estimations of functionals of stochastic processes. Kyiv University, Kyiv, 2008. (in Ukrainian)

[26] Moklyachuk M.P. Minimax-robust estimation problems for stationary stochastic sequences. Stat., Optim. Inf. Comput. 2015, 3 (4), 348-419. doi:10.19139/soic.v3i4.173

[27] Moklyachuk M.P., Golichenko I.I. Periodically correlated processes estimates. LAP Lambert Academic Publishing, Saarbrücken, 2016.

[28] Moklyachuk M.P., Masyutka O.Yu. Robust filtering of stochastic processes. Theory Stoch. Process. 2007, 13 (1-2), 166-181.

[29] Moklyachuk M.P., Masyutka O.Yu. Minimax-robust estimation technique for stationary stochastic processes. LAP Lambert Academic Publishing, Saarbrücken, 2012.

[30] Moklyachuk M.P., Sidei M.I. Interpolation problem for stationary sequences with missing observations. Stat., Optim. Inf. Comput. 2015, 3 (3), 259-275. doi:10.19139/soic.v3i3.149

[31] Moklyachuk M.P., Sidei M. Filtering problem for stationary sequences with missing observations. Stat., Optim. Inf. Comput. 2016, 4 (4), 308-325. doi:10.19139/soic.v4i4.241

[32] Moklyachuk M.P., Sidei M.I. Filtering Problem for functionals of stationary processes with missing observations. Commun. Optim. Theory 2016, Article ID 21, 1-18.

[33] Moklyachuk M.P., Sidei M.I. Extrapolation problem for stationary sequences with missing observations. Stat., Optim. Inf. Comput. 2017, 5 (3), 212-233. doi:10.19139/soic.v5i3.284

[34] Pelagatti M.M. Time series modelling with unobserved components. CRC Press, New York, 2015.

[35] Pourahmadi M., Inoue A., Kasahara Y. A prediction problem in L ${ }^{2}(w)$. Proc. Amer. Math. Soc. 2007,135 (4), 1233-1239. doi:10.1090/S0002-9939-06-08575-3

[36] Pshenichnyj B. N. Necessary conditions of an extremum. Marcel Dekker, New York, 1971.

[37] Rockafellar R. T. Convex Analysis. Princeton University Press, Princeton, 1997.

[38] Rozanov Yu.A. Stationary stochastic processes. Holden-Day, San Francisco-Cambridge-London-Amsterdam, 1967.

[39] Salehi H. Algorithms for linear interpolator and interpolation error for minimal stationary stochastic processes. Ann. Probab. 1979, 7 (5), 840-846.

[40] Vastola S. K., Poor H. V. An analysis of the effects of spectral uncertainty on Wiener filtering. Automatica 1983, 19 (3), 289-293. doi:10.1016/0005-1098(83)90105-X 
[41] Wiener N. Extrapolation, interpolation and smoothing of stationary time series. With engineering applications. The M. I. T. Press, Cambridge, 1966.

[42] Yaglom A.M. Correlation theory of stationary and related random functions. Vol. 1: Basic results. SpringerVerlag, New York etc., 1987.

[43] Yaglom A.M. Correlation theory of stationary and related random functions. Vol. 2: Supplementary notes and references. Springer-Verlag, New York etc., 1987.

Received 24.01.2019

Масютка О. Ю., Моклячук М.П., Сідей М. І. Фільтраиія багатовимірних стаиіонарних послідовностей із пропусками спостережень // Карпатські матем. публ. - 2019. - Т.11, №2. - С. 361-378.

Аосліджується задача оптимального в середньоквадратичному сенсі оцінювання лінійних функціоналів, що залежать від невідомих значень багатовимірних стаціонарних послідовностей. Оцінки базуються на спостереженнях послідовності з адитивним стаціонарним шумом із пропусками спостережень. Знайдено формули для обчислення середньоквадратичних похибок та спектральних характеристик оптимальних оцінок функціоналів у тому випадку, коли спектральні щільності послідовностей точно відома. Мінімаксний (робасиний) метод оцінювання застосовано у тому випадку коли спектральні щільності послідовностей точно невідомі а задані множини допустимих спектральних щільностей. Формули, що визначають найменш сприятливі спектральні щільністі та мінімаксні спектральні характеристики оптимальних оцінок функціоналів, запропоновані для заданих множин допустимих спектральних шільностей.

Ключові слова і фрази: стаціонарні послідовності, мініміксна оцінка, робастна оцінка, середньоквадратична похибка, найменш сприятлива спектральна щільність, мінімаксна спектральна характеристика. 